\title{
Pre-post evaluation of a weight management service for families with overweight and obese children, translated from the efficacious lifestyle intervention Parenting, Eating and Activity for Child Health (PEACH)
}

\author{
Carly J. Moores ${ }^{1 *}$, Jacqueline Miller ${ }^{1,2}$, Lynne A. Daniels ${ }^{3}$, Helen A. Vidgen ${ }^{3}$ and Anthea M. Magarey ${ }^{1}$ \\ ${ }^{1}$ Nutrition and Dietetics, College of Nursing and Health Sciences, Flinders University, GPO Box 2100, Adelaide, SA 5001, Australia \\ ${ }^{2}$ South Australian Health and Medical Research Institute, PO Box 11060, Adelaide, SA 5001, Australia \\ ${ }^{3}$ School of Exercise and Nutrition Sciences, Faculty of Health, Queensland University of Technology, GPO Box 2434, Brisbane, \\ QLD 4001, Australia
}

(Submitted 9 November 2017 - Final revision received 14 March 2018 - Accepted 24 March 2018)

\section{Abstract}

Parenting, Eating and Activity for Child Health (PEACH) is a multi-component lifestyle intervention for families with overweight and obese children. PEACH was translated from an efficacious randomised-controlled trial (RCT) and delivered at scale as PEACH Queensland (QLD) in Queensland, Australia. The aim of this study is to explore pre-post changes in parenting, and child-level eating, activity and anthropometry, in the PEACH QLD service delivery project. PEACH QLD enrolled 926 overweight/obese children (817 families). Pre-programme evaluation was completed for 752 children and paired pre-post-programme evaluation data were available for 388 children. At baseline, children with pre-post-programme data were (mean) 8.8 years old, and at follow-up were 9.3 years old, with mean time between pre-post-programme measures of 0.46 years. Outcomes reflected each domain of the PEACH programme: parenting, eating behaviour of the child and activity behaviours (means reported). Parents reported improvements in parenting self-efficacy $(3.6$ to $3 \cdot 7, P=0 \cdot 001)$. Children had improved eating behaviours: eating more daily serves of vegetables $(2 \cdot 0$ to $2 \cdot 6, P=0 \cdot 001)$ and fewer non-milk sweetened beverages $(0 \cdot 9)$ to $0 \cdot 6, P=0 \cdot 001)$ and discretionary foods $(2 \cdot 2$ to $1 \cdot 5, P=0.001)$. Children spent more time in moderate-to-vigorous physical activity $(86$ to $105 \mathrm{~min} / \mathrm{d}, P=0.001)$ and less time in sedentary screen-based behaviours (190 to $148 \mathrm{~min} / \mathrm{d}, P=0 \cdot 001)$. Consequently, there were significant improvements in mean $\operatorname{BMI} z(-0 \cdot 112 ; P<0 \cdot 001)$ and weight status (healthy weight/overweight/obese/morbidly obese prevalence from $0 / 22 / 33 / 45 \%$ to $2 / 27 / 34$ / $37 \%, P<0.001)$. When delivered at scale, PEACH remains an effective family-based, multi-component, lifestyle weight management programme for overweight and obese children whose families engage in the programme.

Key words: Families: Public health: Translational research: Implementation: Paediatric obesity

Primary and secondary prevention are crucial to tackling the health burden of child obesity and its associated co-morbidities. The World Health Organization Report of the Commission on Ending Childhood Obesity recommends the provision of 'family-based, multi-component, lifestyle weight management services for children and young people who are obese' as part of universal child health care ${ }^{(1)}$. Family-based interventions are central to addressing childhood obesity as parents shape their child's home environment and lifestyle behaviours, including diet and physical activity ${ }^{(2)}$.

Parenting, Eating and Activity for Child Health (PEACH) is a multi-component lifestyle intervention for families with overweight and obese children of primary/elementary school age. The effectiveness of $\mathrm{PEACH}$ has been previously shown in a randomised-controlled trial (RCT) with a clinically and statistically significant $10 \%$ reduction in BMI $z$ at the end of the 6-month intervention that was maintained 18 months later with no further intervention $^{(3)}$. PEACH aims to support parents to modify their child's lifestyle through a whole-of-family approach and is consistent with the Australian Clinical Practice Guidelines for the Management of Overweight and Obesity in Adults, Adolescents and Children ${ }^{(4)}$. The 6-month facilitated group-based programme targets, and is delivered to, parents as the agents of change. A key element of the PEACH programme is parenting and the provision of parental strategies to set limits around lifestyle behaviours as parenting self-efficacy has been inversely associated with parent ability to encourage healthier child behaviours ${ }^{(5)}$.

PEACH Queensland (QLD) received government funding to scale the evidence-based PEACH programme for delivery to families in community settings across the Australian state of Queensland. The call for tender by the Queensland Department of Health to increase the capacity of Queensland families to adopt healthy lifestyles related to healthy eating and physical activity and promote healthy weight and weight management through sustainable behaviour change provided an opportunity

Abbreviations: PEACH, Parenting, Eating and Activity for Child Health; QLD, Queensland; RCT, randomised-controlled trial.

* Corresponding author: Dr C. J. Moores, email carly.moores@flinders.edu.au 
to evaluate the implementation outcomes of PEACH when delivered at scale. The PEACH QLD funder outcomes of interest were about implementation of the programme, and health behaviours pre- and post-programme as a measure of whether benefits of the programme shown previously via RCT remained when the programme was upscaled.

Although efficacy of PEACH and other programmes has been assessed during early stages of development ${ }^{(6)}$, few child obesity management programmes with demonstrated efficacy have been scaled up for implementation in the community or as part of community health services and findings disseminated in the literature ${ }^{(7,8)}$. Implementation and evaluation of efficacious interventions delivered at scale are an important step in translating childhood obesity research into effective management services within universal child health care.

The overall aim of this study is to evaluate outcomes of a multi-component lifestyle programme for families with overweight or obese children delivered in a 'real-world' service/practice setting. PEACH was translated and upscaled from the PEACH intervention previously demonstrated to be efficacious in a research setting. As such, the design of the translational evaluation presented here purposefully does not include a control group.

\section{Methods \\ The Parenting, Eating and Activity for Child Health programme}

PEACH has been described in detail elsewhere ${ }^{(3,9,10)}$, but is briefly guided by six principles: (1) work as a family for children's health; (2) be role models; (3) be balanced and consistent when parenting; (4) base family meals and snacks on the Australian Guide to Healthy Eating ( $\mathrm{AGHE}^{(11)}$ ); (5) be active often and in a variety of ways; and (6) make healthy choices easy choices. Developing parenting self-efficacy is a core component of the programme. Parents are encouraged to lead behaviour change in their families by planning ahead; setting goals; managing behaviour and consequences; and being role models.

\section{Parenting, Eating and Activity for Child Health Queensland}

The Queensland Department of Health awarded a tender to Queensland University of Technology (QUT) in 2013 to deliver PEACH to 1400 children. Stated tender aims were to (1) increase the capacity of the families to adopt healthy lifestyles related to healthy eating and physical activity, and to (2) promote healthy weight and weight management through sustainable behaviour change. The external evaluation (pre- and post-programme design) was subcontracted to researchers led by A. M. M. and J. M. at Flinders University (South Australia) and is described in detail elsewhere ${ }^{(12)}$. The evaluation was approved by Queensland Children's Health Services Human Research Ethics Committee (HREC) (Reference HREC/13/QHC/25); QUT HREC (Reference 1300000633); Flinders University Social and Behavioural Research Ethics Committee (Reference 6231); and Central Queensland University HREC (Reference H13/09-173). Parents provided written, informed consent to participate in this research for themselves and their child. PEACH QLD was registered with the Australian and New Zealand Clinical Trials Registry (ACTRN12617000315314).

Inclusion criteria for children were as follows: (1) residing in Queensland, Australia; (2) above a healthy weight for their age and sex as determined by United States Centers for Disease Control and Prevention (US-CDC) BMI $\geq 85$ th percentile; (3) aged 5-11 years; (4) having a parent with sufficient English to participate in the group sessions; and (5) not concurrently participating in any other lifestyle programme. PEACH was delivered to parents via ten 90-min, face-to-face group sessions by parent facilitators. As in the RCT, sessions one to nine were initially delivered fortnightly (eighteen groups). Subsequently, in response to parent and facilitator feedback, a need to improve engagement and to provide organisational efficiencies, group sessions (for eighty-seven groups) were delivered weekly to align with school terms. This change, and other changes during PEACH QLD implementation, has been previously reported in detail elsewhere ${ }^{(13)}$. Regardless of session frequency, session 10, a reflection and evaluation session, was held approximately 6 months after the start of the programme, with a median of 23 (interquartile range (IQR): 21.5-26.0) weeks. Facilitators aimed to provide three individual phone calls between sessions 9 and 10 to reinforce messages and provide encouragement. While parents attended group sessions, children participated in active and healthy lifestyle activities in separate, concurrent group sessions conducted by two child facilitators. Facilitators were health professionals with relevant experience and received standardised training. Parents could enrol in the programme up to session 3. Pre- and postprogramme evaluation data were collected at the first session attended by the family and at session 10 . Families not attending session 10 were prompted up to three times to complete surveys by email and/or telephone.

\section{Family demographics}

Demographic characteristics including family composition and parent education, country of birth and indigeneity were captured in parent-completed questionnaires. Residential postcodes were used to determine the following: (1) socioeconomic index using the Socio-Economic Indexes for Areas Index of Relative Socio-economic Disadvantage (SEIFA IRSD 2011) ${ }^{(14)}$; and (2) geographic remoteness using the Accessibility/Remoteness Index of Australia (ARIA 2011+) ${ }^{(15)}$. IRSD comprises seventeen measures including low income, low education, high unemployment and level of unskilled occupations. In general, a low IRSD score indicates relatively greater disadvantage, and a high score indicates a relative lack of disadvantage. The ARIA measure of remoteness of Australian areas yields five categories ranging from highly accessible (major cities) to very remote. For the purpose of this paper, parent refers to the supervising adult of children enrolled in PEACH and includes primary caregivers (biological, adoptive and stepparents), as well as other carers (grandparents).

\section{Parent outcomes}

Parenting self-efficacy. Parenting self-efficacy has been inversely associated with parent ability to encourage healthier 
child behaviours ${ }^{(5)}$. Parenting self-efficacy was measured preand post-programme using a four-item questionnaire from the Longitudinal Study of Australian Children ${ }^{(16)}$. Each item enquired about the child's behaviour relative to the parent's desires or the parent's self-perceived ability to manage their child's behaviour. Responses were given on a five-point Likert scale, ranging from 'never/almost never' to 'sometimes' to 'always/almost always' and have been collapsed for reporting. Two items were reverse-scored ${ }^{(17)}$ and an overall parenting self-efficacy score was calculated by averaging the scores from the four items. A higher mean score is indicative of higher parenting self-efficacy.

\section{Child outcomes}

Child anthropometrics. Child weight, height and waist circumference (WC) were measured by the trained child facilitators pre-programme (normally at the first session attended) and post-programme (session 10) using standard procedures and study-provided equipment. In the absence of facilitatormeasured anthropometry data, parent-measured child height and weight were collected where possible (pre-programme $6.9 \%(n$ 52) and post-programme $6.7 \%$ ( $n$ 22)). Age- and sex-standardised $z$ scores were calculated using the USCDC2000 (18) (height $z$, weight $z$, BMI $z$ ) and $\mathrm{UK} 1990^{(19)}$ (waist $z$ ) references. Biologically implausible values for $z$ scores defined by the US-CDC ${ }^{(20)}$ were excluded from analyses, in addition to children who did not have an increase in height from pre- to post-programme. Children were categorised as healthy weight, overweight, obese or morbidly obese using International Obesity Taskforce extended thresholds. A waist: height ratio of $\geq 0.5$ was considered excessive.

Child diet. Parents completed the forty-item semi-quantitative Children's Dietary Questionnaire (CDQ) $)^{(21)}$, and ten-item Core Food Group Intake Tool ${ }^{(22)}$. The previously validated CDQ assesses frequency and variety of intake of foods and food behaviours of particular relevance to children in the context of overweight $^{(22)}$. Four diet quality scores - (1) fruit and vegetables, (2) non-milk sweetened beverages, (3) fat from dairy products and (4) discretionary foods (high fat/high sugar) - are determined from twenty-eight items estimating the variety of fruit and vegetables consumed, frequency of intake of non-milk sweetened beverages (fruit juice/fruit drink and non-diet soft drink/cordial), reduced-fat dairy products and non-core foods high in energy, salt, fat or sugar, respectively. Target scores are based on the Australian Dietary Guidelines ${ }^{(11)}$. The reliability and validity of these scores at the group level has been previously reported ${ }^{(21)}$. A fifth score, food behaviours, uses the remaining twelve items to assess the frequency of behaviours associated with obesity (e.g. eating in front of television, eating meals as a family, size of meals). The Core Food Group Intake Tool was used to determine the number of daily serves of foods from each of the five core food groups in the $\mathrm{AGHE}^{(11)}$ : (1) fruit; (2) vegetables and legumes/beans; (3) grain (cereal) foods, mostly whole grain and/or high cereal fibre varieties; (4) lean meats and poultry, fish, eggs, tofu, nuts and seeds and legumes/beans; and (5) milk, yogurt, cheese and/or alternatives, mostly reduced fat. Examples of common foods in each of the five core food groups, including serve size descriptions, were provided to assist parents completing these questions. Average daily intake was estimated as ((serves on weekdays $\times 5)+($ serves on weekend days $\times 2)) \div 7$. The proportion of children meeting age and sex recommendations ${ }^{(11)}$ for each of these core food groups was determined.

Child physical activity and screen-based sedentary behaviour. Parents reported the length of time their child usually spends in physical activity and screen-based sedentary behaviours in a typical week using the Children's Leisure Activities Study Survey ${ }^{(23)}$. Screen-based sedentary behaviours included watching television/videos, playing Playstation/Nintendo/computer games and using the computer/Internet. These data were used to categorise children in one of two groups - those meeting recommendations and those not meeting recommendations separately for physical activity and sedentary behaviour, based on Australia's Physical Activity and Sedentary Behaviour Guidelines 5-12 Years ${ }^{(24)}$ recommending $\geq 60 \mathrm{~min} / \mathrm{d}$ of moderate-to-vigorous physical activity, and $\leq 2 \mathrm{~h} / \mathrm{d}$ sedentary time, respectively. These physical activity data are available for a subsample as the measurement tool was subsequently modified to reduce participant burden with the aim of improving questionnaire completion.

\section{Data collection, completion and statistical analysis}

Data were collected via survey hardcopy or online completion (SurveyMonkey Inc.). All data were entered, cleaned and analysed in IBM $^{\circledR}$ SPSS $^{\circledR}$ version 23 (IBM Corp.). Continuous data were reported as bootstrapped means and 95\% CI and categorical data were reported as numbers and percentages. Repeated-measures $t$ tests with bootstrapping were used to analyse changes over time for continuous data. Wilcoxon's signed-rank test and related samples McNemar $\chi^{2}$ test was used to assess shifts in categorical variables over time. In keeping with a comparable upscaled programme ${ }^{(8)}$, missing values were not imputed in this study as the objective is to similarly report real-world changes in pre-post evaluation of a service delivery project. The threshold for statistical significance was set at $P<0.05$ and exact two-sided $P$ values are reported.

\section{Sample size}

The PEACH Programme has previously demonstrated efficacy in a RCT of 169 children, of whom $80 \%$ provided data at 6 months ${ }^{(3)}$. There was a $10 \%$ reduction in BMI $z$ for participants in the parenting and healthy lifestyle group ${ }^{(3)}$, which is comparable with the intervention used in the PEACH QLD Project. As PEACH QLD was funded by a service delivery tender from the Queensland Government (Australia), there were no a priori power calculation or sample size analyses. Instead, PEACH QLD had enrolment targets and initially aimed to enrol 1400 children, which was later revised to 1100 children. A total of 1184 children were enrolled in PEACH QLD, of whom 926 were above a healthy weight at baseline. This outcome paper reports the outcomes for this subsample of children. A reduction in the 
effect size of the PEACH intervention was anticipated with the delivery of the intervention at scale in the PEACH QLD Project; however, the magnitude of the difference in effect size between the RCT and large-scale community programme was not able to be estimated a priori owing to a lack of comparable studies in the published literature.

\section{Results}

PEACH QLD enrolled 926 overweight/obese children from families in 105 groups across Queensland, Australia, from October 2013 to September 2016. The level of engagement in providing evaluation data for families and children is shown in Fig. 1. Paired evaluation data (i.e. data provided preprogramme and post-programme) for parenting, diet, activity and anthropometric measures were available for $51 \%, 51,33$ and $44 \%$ of children with pre-programme data, respectively. Owing to variability in questionnaire completion in a real-world service delivery setting, there are varying levels of missing data resulting in differing $n$ values for each evaluation measure.

Table 1 shows characteristics of families in the whole sample who provided any demographic data pre-programme ( $n$ 659). For the purpose of evaluating completion bias, the sample was dichotomised to give two groups: those families who provided pre-programme data only ( $n$ 322) and those who provided paired pre-post-programme data ( $n$ 337). Families with paired pre-post-programme data were more likely be dual-parent households $(P<0 \cdot 001)$, be Australian-born $(P=0 \cdot 011)$ and have higher levels of education $(P=0.002)$ compared with those with pre-programme data only. A comparison of children with paired pre-post $v$. pre-programme data only indicates that those with paired data were younger $(-0.29$ years; $95 \%$ CI -0.54 , -0.03) $(P=0.029)$, and appeared to be proportionately more female ( 57 to $52 \%$ ), with a lower BMI $z$ score $(-0 \cdot 039$; $95 \%$ CI $-0 \cdot 1,0 \cdot 026)$; however, neither of the latter differences reached statistical significance. At baseline, children with pre-post-programme data were 8.8 (95\% CI 8.6, 9.0) years old, and at follow-up were $9 \cdot 3$ (95\% CI 9.1, 9.4) years old, with the mean time between pre- and post-programme measures being 0.46 (95\% CI $0.45,0.47$ ) years (approximately 5.5 months). In all, $22 \%$ ( $n$ 206) of enrolled families did not attend any sessions. Overall, for families who attended at least one session ( $n$ 618; 720 children), median attendance was 7 (IQR 3-9) of a maximum ten sessions, whereas families providing paired data ( $n 337$ families; $n 388$ children) attended 8 (IQR 7-9) of ten sessions.

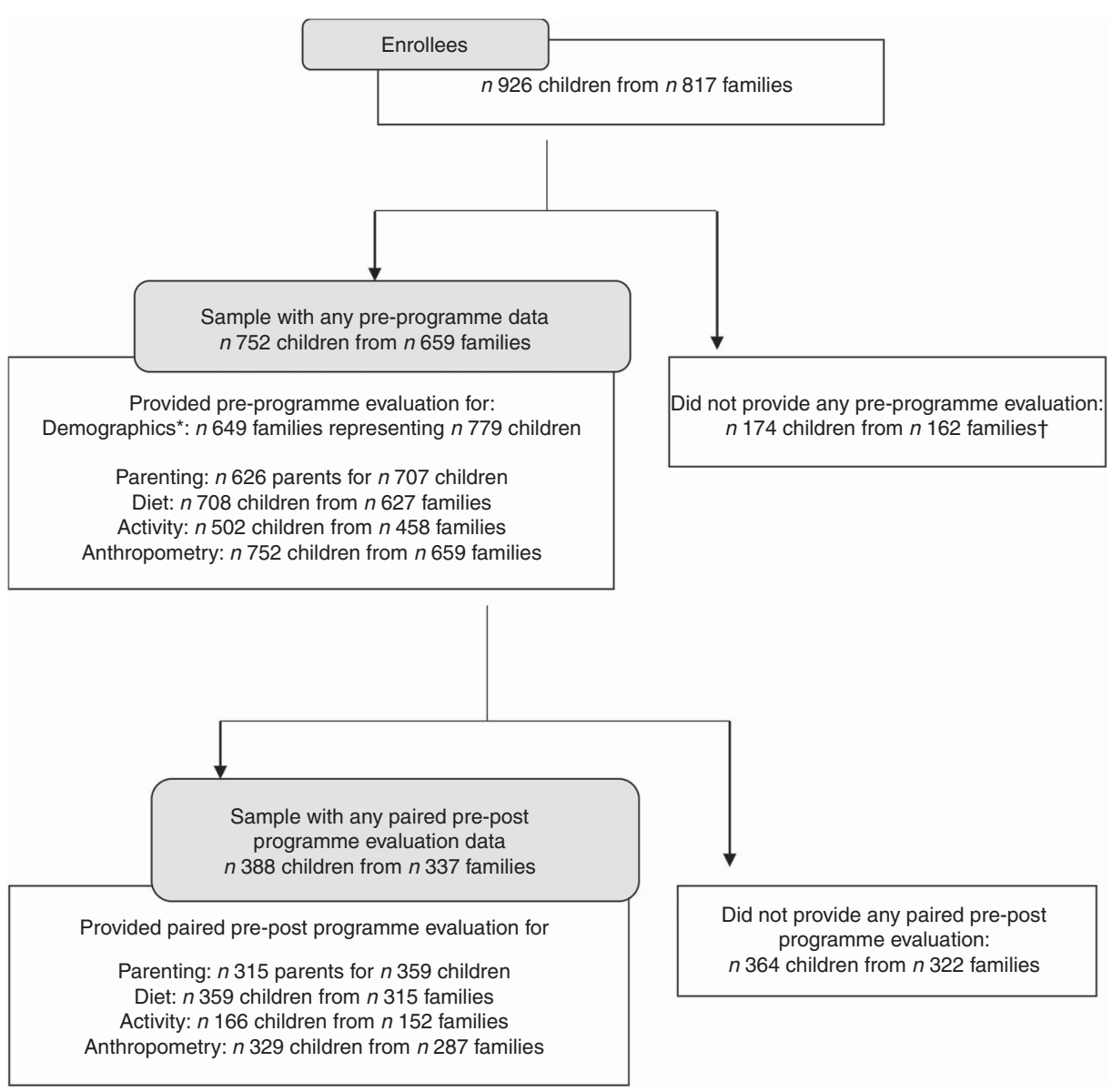

Fig. 1. Flow chart of data collected in Parenting, Eating and Activity for Child Health Queensland. * Parent and family demographic data are collected at the family level, and not for each child. Hence, some children may have parent- and family level demographics but no child-level data (e.g. diet, anthropometry). † Families with more than one enrolled child may have had children with and without child-level baseline data. 
Table 1. Pre-programme demographics of families, parents and children in Parenting, Eating and Activity for Child Health Queensland (Numbers and percentages; mean values and $95 \%$ confidence intervals)

\begin{tabular}{|c|c|c|c|c|c|c|c|c|c|c|}
\hline & \multicolumn{3}{|c|}{$\begin{array}{l}\text { Whole sample with any } \\
\text { pre-programme data }\end{array}$} & \multicolumn{3}{|c|}{$\begin{array}{l}\text { Sample with pre- } \\
\text { programme data only }\end{array}$} & \multicolumn{3}{|c|}{$\begin{array}{l}\text { Sample with paired pre-post- } \\
\text { programme data for any outcome }\end{array}$} & \multirow[b]{3}{*}{$P^{*}$} \\
\hline & \multirow[b]{2}{*}{ Total $(n)$} & \multicolumn{2}{|c|}{ Value } & \multirow[b]{2}{*}{ Total $(n)$} & \multicolumn{2}{|c|}{ Value } & \multirow[b]{2}{*}{ Total $(n)$} & \multicolumn{2}{|c|}{ Value } & \\
\hline & & $n$ & $\%$ & & $n$ & $\%$ & & $n$ & $\%$ & \\
\hline \multicolumn{11}{|l|}{ Family } \\
\hline \multicolumn{11}{|l|}{ Family SEIFA (IRSD quintile) } \\
\hline 1 (relatively greater disadvantage in general) & 659 & 76 & 12 & 322 & 42 & 13 & 337 & 34 & 10 & 0.564 \\
\hline 2 & & 116 & 18 & & 55 & 17 & & 61 & 18 & \\
\hline 3 & & 127 & 19 & & 64 & 20 & & 63 & 19 & \\
\hline 4 & & 233 & 35 & & 115 & 36 & & 118 & 35 & \\
\hline 5 (relative lack of disadvantage in general) & & 107 & 16 & & 46 & 14 & & 61 & 18 & \\
\hline \multicolumn{11}{|l|}{ Family ARIA } \\
\hline Highly accessible $(0-0.2)$ & 659 & 468 & 71 & 322 & 224 & 70 & 337 & 244 & 72 & 0.844 \\
\hline Accessible $(>0 \cdot 2-2 \cdot 4)$ & & 67 & 10 & & 33 & 10 & & 34 & 10 & \\
\hline Moderately accessible (>2.4-5.92) & & 90 & 14 & & 47 & 15 & & 43 & 13 & \\
\hline Remote/very remote $(>5.92-15)$ & & 34 & 5 & & 18 & 6 & & 16 & 5 & \\
\hline \multicolumn{11}{|l|}{ Parent† } \\
\hline \multirow{2}{*}{\multicolumn{11}{|c|}{ Parent relationship status }} \\
\hline & & & & & & & & & & \\
\hline Partnered (married/de facto) & 628 & 457 & 73 & 296 & 191 & 65 & 332 & 266 & 80 & $<0.001$ \\
\hline Un-partnered (single/separated/divorced/widowed) & & 171 & 27 & & 105 & 36 & & 66 & 20 & \\
\hline \multicolumn{11}{|l|}{ Parent indigeneity and place of birth } \\
\hline Aboriginal and/or Torres Strait Islander & 628 & 30 & 5 & 295 & 16 & 5 & 333 & 14 & 4 & 0.511 \\
\hline Australian-born & & 494 & 79 & & 219 & 74 & & 275 & 83 & 0.011 \\
\hline \multicolumn{11}{|l|}{ Parent education } \\
\hline$<$ Year 12 & 627 & 104 & 17 & 294 & 63 & 21 & 333 & 41 & 12 & 0.002 \\
\hline Year 12 & & 97 & 16 & & 48 & 16 & & 49 & 15 & \\
\hline Trade/apprenticeship/college certificate & & 238 & 38 & & 112 & 38 & & 126 & 38 & \\
\hline University degree & & 188 & 30 & & 71 & 24 & & 117 & 35 & \\
\hline \multicolumn{11}{|l|}{ Household composition } \\
\hline Two parents & 628 & 466 & 74 & 296 & 197 & 67 & 332 & 269 & 81 & $<0.001$ \\
\hline One parent only/50:50 split care & & 129 & 21 & & 82 & 28 & & 47 & 14 & \\
\hline One or two parents with other adults & & 33 & 5 & & 17 & 6 & & 16 & 5 & \\
\hline Children spend time living in other households & 625 & 104 & 17 & 295 & 58 & 20 & 330 & 46 & 14 & 0.067 \\
\hline All care providers supportive of programme & 620 & 580 & 94 & 292 & 271 & 93 & 328 & 309 & 94 & 0.515 \\
\hline \multicolumn{11}{|l|}{ Child $\ddagger$} \\
\hline Age (years) & 752 & & & 364 & \multirow{2}{*}{\multicolumn{2}{|c|}{$9 \cdot 1$}} & 388 & & & 0.029 \\
\hline Mean & \multicolumn{3}{|c|}{8.9} & & & & & & & \\
\hline $95 \% \mathrm{Cl}$ & & $8 \cdot 8$ & & & $8 \cdot 9$ & & & & & \\
\hline Sex (female) & 752 & 410 & 55 & 364 & 188 & 52 & 388 & 222 & 57 & 0.143 \\
\hline BMI $z$ score & 752 & & & 364 & & & 388 & & & 0.226 \\
\hline Mean & & & & & 2. & & & & & \\
\hline $95 \% \mathrm{Cl}$ & & $2 \cdot 18$ & & & 2.19, & 27 & & $2 \cdot 1$ & & \\
\hline International Obesity Task Force weight status & & & & & & & & & & \\
\hline Overweight & 752 & 161 & 21 & 364 & 75 & 21 & 388 & 86 & 22 & 0.903 \\
\hline Obese & & 237 & 32 & & 111 & 31 & & 126 & 33 & \\
\hline Morbidly obese & & 354 & 47 & & 178 & 49 & & 176 & 45 & \\
\hline
\end{tabular}

SEIFA IRSD, socio-economic index for areas index of relative socio-economic disadvantage; ARIA, Accessibility/Remoteness Index for Areas.

${ }^{\star} P$ reports statistical difference between sample with pre-programme data only and sample with both pre- and post-programme paired data.

† Parent demographics are reported at the family level.

$\ddagger$ United States Centers for Disease Control and Prevention reference for BMl $z^{(18)}$; extended International Obesity Task Force weight status cut-off points ${ }^{(25)}$.

\section{Anthropometry}

Distribution of children across weight status categories and $z$ scores pre-programme, and for the sample with paired pre-post-programme data, are shown in Table 2. After intervention there were significant decreases in BMI $z(-0 \cdot 11$, $P<0.001)$ and waist $z(-0.05, P=0.012)$. The prevalence of children classified as morbidly obese declined by 8 percentage points, corresponding to percentage point increases in prevalence of children classified as healthy weight, overweight and obese: 2,5 and $1 \%$, respectively $(P<0 \cdot 001)$.

\section{Diet}

Pre-programme and paired pre-post-programme changes in diet for both core foods and CDQ scores are shown in Table 3. There was a significant improvement in the proportion of children meeting age and sex recommendations ${ }^{(11)}$ for serves of foods from the vegetables and legumes/beans core food group ( $4 v .9 \%, P=0.001$ ). There was a reduction in the proportion of children meeting the daily serve recommendation for cereal (grain) foods from 27 to $21 \%(P=0.024)$, corresponding to a mean decrease of a third of a serve. There were significant 
Table 2. Anthropometry for children in Parenting, Eating and Activity for Child Health Queensland (Numbers and percentages; mean values and $95 \%$ confidence intervals)

\begin{tabular}{|c|c|c|c|c|c|c|c|c|c|c|c|c|c|}
\hline \multirow[b]{3}{*}{ Measure (units) $\dagger$} & & & & & & \multicolumn{8}{|c|}{ Sample with pre-post-programme paired data } \\
\hline & \multicolumn{5}{|c|}{ Pre-programme } & \multicolumn{3}{|c|}{ Pre-programme } & \multicolumn{2}{|c|}{ Post-programme } & \multicolumn{2}{|c|}{ Mean difference } & \multirow[b]{2}{*}{$P^{*}$} \\
\hline & Total $(n)$ & $n$ & $\%$ & Mean & $95 \% \mathrm{Cl}$ & Total $(n)$ & Mean $/ n$ & $95 \% \mathrm{Cl} / \%$ & Mean/n & $95 \% \mathrm{Cl} / \%$ & Mean $/ \Delta \%$ & $95 \% \mathrm{Cl}$ & \\
\hline BMI $\left(\mathrm{kg} / \mathrm{m}^{2}\right) \ddagger$ & 752 & - & & $26 \cdot 4$ & $26 \cdot 0,26 \cdot 7$ & 326 & $25 \cdot 7$ & $25 \cdot 2,26 \cdot 2$ & 25.6 & $25 \cdot 1,26 \cdot 0$ & -0.18 & $-0.33,-0.04$ & 0.015 \\
\hline Height $z$ score & 751 & - & & 1.08 & $1.01,1 \cdot 16$ & 327 & 1.01 & $0.91,1.11$ & 1.05 & $0.95,1.15$ & 0.041 & $0.020,0.064$ & $<0.001$ \\
\hline Weight $z$ score & 751 & - & & 2.34 & $2 \cdot 29,2.38$ & 328 & $2 \cdot 28$ & $2 \cdot 20,2 \cdot 23$ & $2 \cdot 18$ & $2 \cdot 11,2 \cdot 26$ & -0.093 & $-0.115,-0.073$ & $<0.001$ \\
\hline BMl z score & 752 & - & & $2 \cdot 21$ & $2 \cdot 18,2 \cdot 24$ & 326 & $2 \cdot 18$ & $2 \cdot 14,2 \cdot 23$ & 2.07 & $2 \cdot 02,2 \cdot 12$ & -0.112 & $0.090,0.138$ & $<0.001$ \\
\hline Waist $z$ score & 699 & - & & 3.47 & $3.41,3.52$ & 299 & 3.43 & $3.35,3.50$ & 3.38 & $3.30,3.44$ & -0.049 & $-0.084,-0.009$ & 0.012 \\
\hline WHtR & 699 & - & & 0.62 & $0.62,0.63$ & 299 & 0.61 & $0.61,0.62$ & 0.61 & $0.60,0.62$ & -0.005 & $-0.009,-0.0006$ & 0.024 \\
\hline WHtR $(<0.5)$ & 699 & 19 & 3 & & - & 299 & 7 & 2 & 13 & 4 & & $\uparrow 2$ & 0.146 \\
\hline Healthy weight & 752 & - & & & - & 326 & & - & 6 & 2 & & $\uparrow 2$ & $<0.001$ \\
\hline Overweight & & 161 & 21 & & - & & 73 & 22 & 89 & 27 & & $\uparrow 5$ & \\
\hline Obese & & 237 & 32 & & - & & 108 & 33 & 112 & 34 & & $\uparrow 1$ & \\
\hline Morbidly obese & & 354 & 47 & & - & & 145 & 45 & 119 & 37 & & $\downarrow 8$ & \\
\hline
\end{tabular}

WHtR, waist:height ratio.

*Paired-samples $t$ test, McNemar $x^{2}$ test or Wilcoxon's signed-rank test used; $P$ indicates difference between pre- and post-programme paired data.

† United States Centers for Disease Control and Prevention references ${ }^{(18)}$ for heightz, weight $z$ and BMI $z$, UK1990 ${ }^{(19)}$ reference for waist $z$; extended International Obesity Task Force weight status cut-off points ${ }^{(25)}$; WHtR $>0.5$ considered excessive ${ }^{(26)}$.

‡ One child only BMI was available from hospital records (no separate height and weight).

improvements in all CDQ scores post-programme, indicating changes in consumption and behaviours in line with nutrition objectives for the programme, which are based on child diet recommendations.

\section{Physical activity and screen-based sedentary behaviour}

Time spent engaged in physical activity and screen-based sedentary behaviour and the proportion meeting recommendations are described in Table 4, for the subsample for whom pre-post data were available ( $n$ 166). There was a mean increase of $19 \mathrm{~min}$ of physical activity per d (from 86 to $105 \mathrm{~min} / \mathrm{d}$, $P=0.001)$, corresponding to a $9 \%$ increase in children meeting this recommendation at the end of the programme (64v.73\%, $P=0 \cdot 017)$. The proportion of children meeting sedentary activity guidelines also increased (34 v. 50\%, $P=0.001$ ) consistent with the significant decrease of $42 \mathrm{~min}$ of sedentary screen time per $\mathrm{d}$ (190 v. 148, $P=0.001)$.

\section{Parenting self-efficacy}

Although children were the enrollees in PEACH, the intervention was delivered to parents. Table 5 reports the changes in parenting self-efficacy. Parents reported improvements in the following three domains of parenting self-efficacy. Does your child behave in a manner different from the way you want him/ her to? Do you feel that you are good at getting this child to do what you want him/her to do? Do you feel that you are in control and on top of things when you are caring for your child? No significant changes were found in the domain 'Do you think that your child's behaviour is more than you can handle?' This corresponded to a significant increase in overall parenting selfefficacy score (3.6 to $3 \cdot 7$, of a maximum $5 \cdot 0, P=0.001$ ).

\section{Summary of outcomes}

Table 6 reports all outcomes from PEACH QLD and whether there was a positive change (improved), no statistically significant change or a negative change (worsened) in parenting, health behaviours and anthropometry.

\section{Discussion}

A growing body of literature suggests that parent-led interventions for child obesity management, or those that target family rather than individual child behaviours, are effective ${ }^{(27,28)}$. Here we demonstrate that PEACH delivered to parents of obese primary school-aged children improved parent self-efficacy that in turn was consistent with favourable changes in child diet, with increases in daily serves of vegetables, variety and frequency of fruit and vegetable intake and decreases in the consumption of discretionary foods and non-milk sweetened beverages. In additionally, there were desirable changes in activity patterns with increased time spent in physical activity and decreased levels of screen-based sedentary behaviour. Overall, the stated tender aims related to enhancing capacity of families to adopt healthy lifestyles were met.

These lifestyle changes are consistent with reductions in both BMI $z$ scores and the prevalence of obesity and overweight, indicating that PEACH remains effective when delivered at scale. Despite the fact that there was an $8 \%$ reduction in the prevalence of morbid obesity and reduction in $\mathrm{BMI} z$, at the end of the programme most children remained above a healthy weight. Hence, ongoing maintenance of lifestyle changes is required for long-term improvements in child weight status. PEACH provides parents with skills and knowledge to make sustainable lifestyle changes, and at the end of the programme parents are encouraged to continue to apply the programme principles, and these messages are reinforced at the final session, and in personalised feedback letters to families upon programme completion.

We have previously shown in an RCT that PEACH is efficacious with approximately $10 \%$ relative weight reduction at 6 months $^{(3)}$. In the present study, we observed an approximate $5 \%$ reduction in BMI $z$ representing a halving of effect when delivered at scale. This effect size is similar to that shown in a 
Table 3. Diet intake and food behaviour for children in Parenting, Eating and Activity for Child Health Queensland*

(Numbers and percentages; mean values and $95 \%$ confidence intervals)

\begin{tabular}{|c|c|c|c|c|c|c|c|c|c|c|c|c|c|c|c|c|c|c|c|c|}
\hline \multicolumn{21}{|c|}{ Australian Guide to Healthy Eating (AGHE) recommended daily serves of core foods ${ }^{(11)}$} \\
\hline & \multicolumn{11}{|c|}{ Serves per day } & \multicolumn{9}{|c|}{ Meeting recommended serves per day } \\
\hline & & & & & & Sampl & le with & pre-post-pr & rogrami & me paired de & & & & & Sample & with & $\begin{array}{l}\text { pre-1 } \\
\text { aired }\end{array}$ & $\begin{array}{l}\text {-post }-p \\
\text { d data }\end{array}$ & -prog & ramme \\
\hline & & & \multicolumn{3}{|c|}{ Pre-programme } & \multirow[b]{2}{*}{ Total $(n)$} & \multicolumn{2}{|c|}{ Pre-programme } & \multicolumn{2}{|c|}{ Post-programme } & \multirow[b]{2}{*}{$P$} & \multicolumn{3}{|c|}{ Pre-programme } & \multirow[b]{2}{*}{ Total $(n)$} & \multicolumn{2}{|c|}{ Pre } & \multicolumn{2}{|c|}{ Post } & \multirow[b]{2}{*}{$P$} \\
\hline \multicolumn{3}{|l|}{ AGHE core food group } & Total $(n)$ & Mean & $95 \% \mathrm{Cl}$ & & Mean & $95 \% \mathrm{Cl}$ & Mean & $95 \% \mathrm{Cl}$ & & Total $(n)$ & $n$ & $\%$ & & $n$ & $\%$ & $n$ & $\%$ & \\
\hline \multicolumn{3}{|l|}{ Fruit } & 683 & 1.9 & $1 \cdot 8,2 \cdot 0$ & 337 & $2 \cdot 0$ & $1 \cdot 9,2 \cdot 2$ & $2 \cdot 0$ & $1 \cdot 9,2 \cdot 1$ & 0.966 & 683 & 380 & 56 & 337 & 199 & 59 & 213 & 63 & 0.136 \\
\hline \multirow{2}{*}{\multicolumn{3}{|c|}{$\begin{array}{l}\text { Vegetables and legumes/beans } \\
\text { Grain (cereal) foods, mostly whole grain and/or high cereal } \\
\text { fibre varieties }\end{array}$}} & 679 & 1.9 & $1 \cdot 8,2 \cdot 0$ & 337 & $2 \cdot 0$ & $1 \cdot 9,2 \cdot 1$ & $2 \cdot 6$ & $2 \cdot 5,2 \cdot 8$ & 0.001 & 679 & 22 & 3 & 337 & 12 & 4 & 29 & 9 & 0.001 \\
\hline & & & 679 & $2 \cdot 8$ & $2 \cdot 7,3 \cdot 0$ & 334 & $3 \cdot 1$ & $2 \cdot 9,3 \cdot 3$ & $2 \cdot 8$ & $2 \cdot 7,3 \cdot 0$ & 0.042 & 679 & 159 & 23 & 334 & 91 & 27 & 70 & 21 & 0.024 \\
\hline \multicolumn{3}{|c|}{$\begin{array}{l}\text { Lean meats and poultry, fish, eggs, tofu, nuts and seeds and } \\
\text { legumes/beans }\end{array}$} & 680 & $1 \cdot 7$ & $1 \cdot 6,1 \cdot 8$ & 332 & $1 \cdot 7$ & $1 \cdot 6,1 \cdot 8$ & $1 \cdot 7$ & $1 \cdot 7,1 \cdot 8$ & 0.741 & 680 & 226 & 33 & 332 & 116 & 35 & 105 & 32 & 0.334 \\
\hline \multicolumn{3}{|c|}{ Milk, yogurt, cheese and/or alternatives, mostly reduced fat } & 681 & $2 \cdot 0$ & $1 \cdot 9,2 \cdot 1$ & 332 & $2 \cdot 1$ & $2 \cdot 0,2 \cdot 2$ & $2 \cdot 0$ & $1 \cdot 9,2 \cdot 1$ & 0.169 & 681 & 285 & 42 & 332 & 159 & 48 & 149 & 45 & 0.373 \\
\hline \multicolumn{21}{|c|}{ Children's Dietary Questionnaire $(\mathrm{CDQ})^{(21)}$} \\
\hline \multirow[b]{4}{*}{ CDQ subscale } & \multirow[b]{4}{*}{ Range } & \multirow[b]{4}{*}{ Target } & \multicolumn{9}{|c|}{ Score } & \multicolumn{9}{|c|}{ Meeting target score } \\
\hline & & & \multicolumn{9}{|c|}{ Sample with pre-post-programme paired data } & \multicolumn{9}{|c|}{$\begin{array}{l}\text { Sample with pre-post-programme } \\
\text { paired data }\end{array}$} \\
\hline & & & Pre & -progra & $\mathrm{mme}$ & & Pre-pr & rogramme & Post-p & orogramme & \multirow[b]{2}{*}{$P$} & Pre-prog & gramm & & \multirow[b]{2}{*}{ Total $(n)$} & \multicolumn{2}{|c|}{ Pre } & Po & ost & \\
\hline & & & Total $(n)$ & Mean & $95 \% \mathrm{Cl}$ & Total $(n)$ & Mean & $95 \% \mathrm{Cl}$ & Mean & $95 \% \mathrm{Cl}$ & & Total $(n)$ & $n$ & $\%$ & & $n$ & $\%$ & $n$ & $\%$ & $P$ \\
\hline Fruit and vegetables $\dagger$ & $0-28$ & $\geq 18$ & 692 & $12 \cdot 3$ & $11 \cdot 9,12 \cdot 7$ & 347 & $13 \cdot 1$ & $12 \cdot 5,13 \cdot 7$ & 14.2 & $13 \cdot 7,14.8$ & 0.001 & 692 & 100 & 15 & 347 & 67 & 19 & 76 & 22 & 0.362 \\
\hline Non-milk sweetened beverages $\ddagger$ & $0-6$ & $\leq 1$ & 697 & 1.0 & $0.9,1 \cdot 1$ & 351 & 0.9 & $0.8,1 \cdot 0$ & 0.6 & $0.5,0.7$ & 0.001 & 697 & 450 & 65 & 351 & 241 & 69 & 274 & 78 & 0.001 \\
\hline Fat from dairy products $\ddagger$ & $0-15$ & $\leq 0.28$ & 682 & $3 \cdot 2$ & $3 \cdot 0,3 \cdot 4$ & 328 & $3 \cdot 2$ & $3.0,3.5$ & $2 \cdot 8$ & $2 \cdot 6,3 \cdot 0$ & 0.001 & 682 & 50 & 7 & 328 & 22 & 7 & 32 & 10 & 0.134 \\
\hline Discretionary foods (high fat/high sugar)‡ & $0-10 \cdot 3$ & $\leq 2$ & 676 & $2 \cdot 2$ & $2 \cdot 1,2 \cdot 2$ & 320 & $2 \cdot 2$ & $2 \cdot 1,2 \cdot 4$ & 1.5 & $1.4,1.6$ & 0.001 & 676 & 338 & 50 & 320 & 157 & 49 & 233 & 73 & $<0.001$ \\
\hline Food behaviours $\dagger$ & $2-54$ & $\geq 42$ & 684 & $31 \cdot 0$ & $30 \cdot 1,31 \cdot 2$ & 335 & $30 \cdot 9$ & $30 \cdot 3,31 \cdot 6$ & 35.7 & $35 \cdot 1,36 \cdot 3$ & 0.001 & 684 & 14 & 2 & 335 & 7 & 2 & 47 & 14 & $<0.001$ \\
\hline
\end{tabular}

${ }^{*} 708$ children had any diet data pre-programme (from 676 to 683 for individual outcomes) and 359 children had any paired pre-post-programme diet data (from 320 to 351 for individual outcomes). Paired-samples $t$ test used for continuous data; related samples McNemar $x^{2}$ test used for categorical data.

$\dagger$ A higher score indicates a healthier intake/behaviour.

₹ A lower score indicates a healthier intake. 
Table 4. Time spent by the child in screen-based sedentary behaviours and moderate-to-vigorous physical activity* (Numbers and percentages; mean values and $95 \%$ confidence intervals)

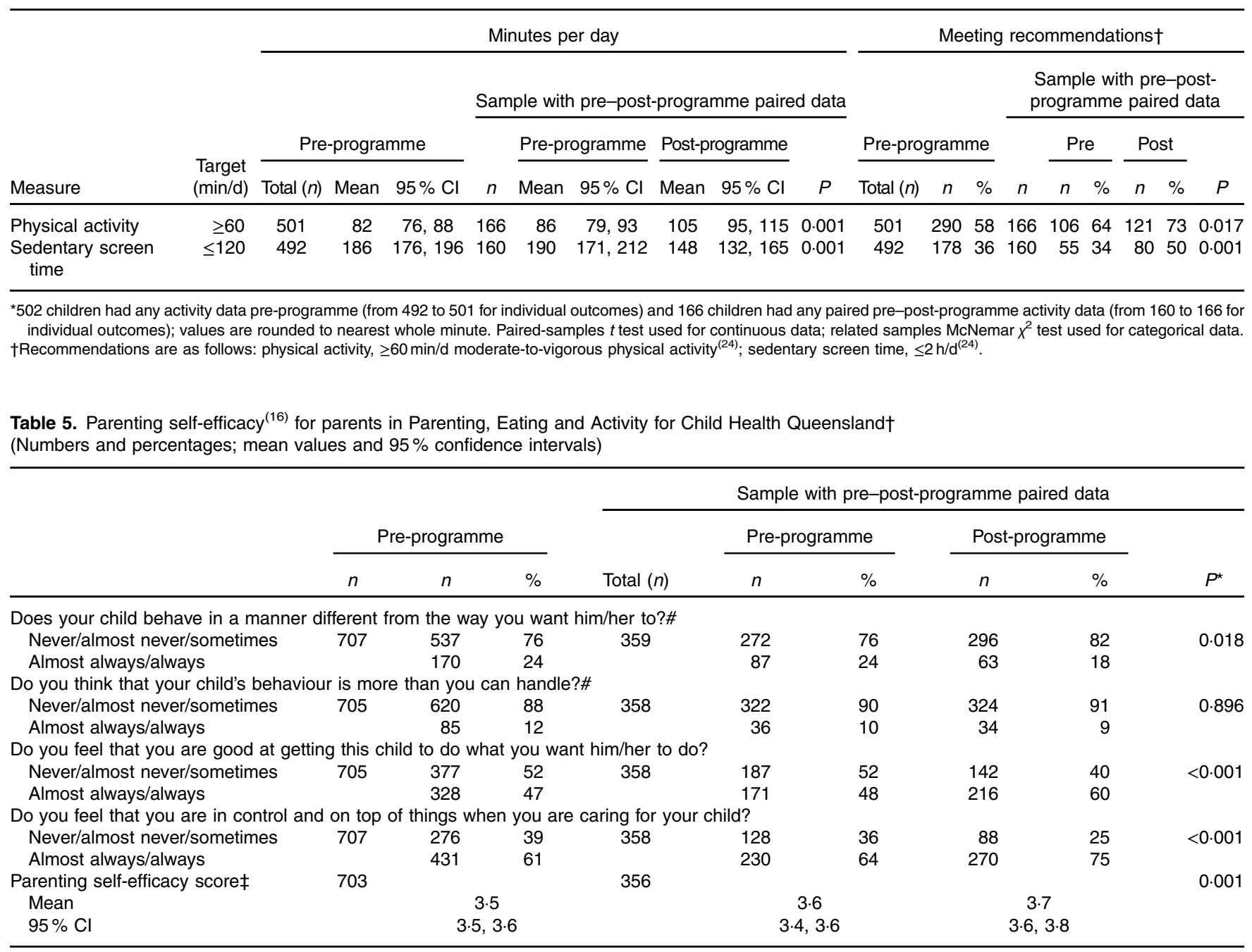

*Paired-samples $t$ test used for continuous data; related samples McNemar $x^{2}$ test used for categorical data.

$\dagger 707$ children had any parenting self-efficacy data pre-programme (from 705 to 707 for individual outcomes) and 359 children had any pre-post-programme paired parenting selfefficacy data (from 358 to 359 for individual outcomes).

$\ddagger$ Combined score reports mean of all four items (Cronbach's a 0.754 ) with items 1 and 2 (marked \#) reverse scored ${ }^{(16)}$.

recent study of children ( $n$ 1776) who participated in the 10-week Go4Fun programme (approximately 5\%, NSW, Australia), which was also approximately half the effect reported in the original efficacy trial that reported outcomes at 6 months ${ }^{(8,29)}$. The Go4Fun was adapted from Mind, Exercise, Nutrition..Do it!, which had been previously delivered at scale in the UK and reported to yield a relative weight loss of $6 \cdot 7 \%{ }^{(7)}$. Not surprisingly, it appears that the relative weight loss that can be achieved when a parent-led child obesity programme is scaled for health service delivery will be less than that observed in more rigorous, tightly controlled RCT. Notwithstanding this reduction in effect size, we have here demonstrated effectiveness of PEACH when delivered on a larger scale as a community health programme. Although it is unlikely that any single programme can solve the complex issue of childhood obesity in isolation, the evidence-based PEACH intervention should be included within a multi-strategic approach to its prevention, which includes supporting children who are already overweight or obese ${ }^{(30)}$.

PEACH has a specific focus on parenting skills, encouraging an authoritative parenting style and supporting parents to be agents of change and role models for their child's health behaviours. Although there was a statistically significant improvement in parenting self-efficacy score from pre- to postprogramme, the magnitude of the change in the score derived from four items is notably small. However, our results show that post programme fewer parents reported their child behaving in a manner different from that they want them to, and more parents reported feeling good at getting their child to do what they want them to, and feeling in control and on top of things when caring for their child. Other studies identified in a recent Cochrane review of parent-only interventions for childhood overweight or obesity in children aged 5-11 years ${ }^{(28)}$ reported inconsistent measures of parenting, which cannot be compared 
Table 6. Summary table of outcomes in Parenting, Eating and Activity for Child Health Queensland

\begin{tabular}{|c|c|c|c|c|c|}
\hline $\begin{array}{l}\text { PEACH } \\
\text { domain }\end{array}$ & Measure (level)† & Improved & $\begin{array}{c}\text { No } \\
\text { changeł }\end{array}$ & Worsened & $\begin{array}{l}\text { For detailed } \\
\text { results refer to }\end{array}$ \\
\hline P Parenting & Parenting self-efficacy $(p)$ & $J^{* \star}$ & & & Table 5 \\
\hline \multirow[t]{10}{*}{ E Eating } & Daily serves: fruit (c) & & $\checkmark$ & & Table 3 \\
\hline & Daily serves: vegetables and legumes/beans (c) & $J^{\star *}$ & & & \\
\hline & Daily serves: grain (cereal) foods, mostly whole grain and/or high cereal fibre varieties (c) & & & $\mathfrak{J}^{*}$ & \\
\hline & Daily serves: lean meats and poultry, fish, eggs, tofu, nuts and seeds and legumes/beans (c) & & $\checkmark$ & & \\
\hline & Daily serves: milk, yogurt, cheese and/or alternatives, mostly reduced fat (c) & & $\checkmark$ & & \\
\hline & Diet quality score: fruit and vegetables (c) & $J^{\star \star}$ & & & \\
\hline & Diet quality score: non-milk sweetened beverages (c) & $J^{* *}$ & & & \\
\hline & Diet quality score: fat from dairy products (c) & $J^{\star \star}$ & & & \\
\hline & Diet quality score: discretionary foods (high fat/high sugar) (c) & $J^{* *}$ & & & \\
\hline & Diet quality score: food behaviour (c/f) & $J^{* *}$ & & & \\
\hline \multirow[t]{2}{*}{ A Activity } & Physical activity (c) & $\sigma^{\star *}$ & & & Table 4 \\
\hline & Sedentary behaviour (c) & $J^{\star *}$ & & & \\
\hline \multirow{6}{*}{$\begin{array}{l}\text { C Child } \\
\text { H Health }\end{array}$} & $\mathrm{BMI}(\mathrm{c})$ & $J^{\star \star}$ & & & Table 2 \\
\hline & $\mathrm{BMI} z(\mathrm{c})$ & $J^{\star \star}$ & & & \\
\hline & Weightz (c) & $J^{* *}$ & & & \\
\hline & Weight status (c) & $J^{* *}$ & & & \\
\hline & Waistz (c) & $J^{* *}$ & & & \\
\hline & $\mathrm{WHtR}(\mathrm{c})$ & $J^{* *}$ & & & \\
\hline
\end{tabular}

WHtR, waist:height ratio.

* $P<0.05,{ }^{* \star} P<0.01$.

$\dagger(\mathrm{c})$, Child; (f), family; (p), parent.

$\ddagger$ No statistically significant change.

with each other or the present study (Parenting Scale ${ }^{(31)}$, Alabama Parenting Questionnaire ${ }^{(3)}$ and Ghent Parental Behaviour Scale $\left.{ }^{(32)}\right)$. In addition, unlike PEACH, not all familybased child obesity programmes have a strong emphasis on parenting. Future research is needed to further understand how parenting skills training and practices can affect child and family healthy lifestyle behaviours including whether there is any interactive effect with more than one parent/caregiver, domainspecific parenting behaviours ${ }^{(33)}$ and the sustainability of parenting changes in the long term, including its association with child lifestyle behaviours.

Family engagement was challenging with approximately one in five enrolled families not providing any data and not attending any sessions. The characteristics of this group cannot be described owing to the lack of available data. Demographics associated with PEACH QLD attendance have been reported elsewhere: briefly, advantaged families, parents who were partnered, with higher education levels and those who selfreferred to PEACH had higher programme attendance ${ }^{(34)}$. Of the families who provided pre-programme data, approximately half also provided post-programme data allowing pre-postprogramme evaluation of effectiveness. Children with paired pre-post-programme data tended to come from families of slightly higher SES and generally had more favourable diet and activity behaviours initially than children with pre-programme data only. Overall, as expected, engagement and retention bias were evident and potentially limit the generalisability of the programme outcomes for all families with children above a healthy weight range. The relationship between disadvantage and health is complex ${ }^{(35)}$, and although disadvantaged and minority groups have a higher burden of child obesity, engagement of these populations in PEACH was limited. This suggests that PEACH may be better suited to more highly educated, double-parent families, or as a result of their relative advantage such families may have greater resources to improve their capacity to participate and attend programme sessions.

Although attrition from the programme was not directly reported here, evaluation data provision is a reasonable proxy for attrition as anthropometric measurements were taken in the final session. Attrition rates reported in the literature vary widely, and depend on the definition ${ }^{(36,37)}$. A recent review of twenty-three studies reported a median attrition rate of $37 \%$ (ranging from 4 to $83 \%$ ) from paediatric obesity management services ${ }^{(36)}$. This review also found that older children were more likely to discontinue care; however, sex and preprogramme weight status did not predict treatment attrition. Our findings were similar with respect to age, as children with paired data were younger, but we also found evidence of differences in sex (higher proportion of females) and weight status (lower mean $\mathrm{BMI} z$ ) for those children with paired data. The overall higher proportion of girls is in line with the PEACH $\mathrm{RCT}^{(3)}$ and unsurprising, as previous studies have found parents of overweight girls more frequently recognised as being overweight, compared with boys ${ }^{(38,39)}$. The proportion of girls was then greater in the sample providing pre-post-programme data, which may indicate that parents of girls rate their need to participate in the programme, and/or its benefits to their child's health, higher than parents of boys or that girls are more weight conscious and willing to seek/continue treatment. In addition, the over-representation of obese and morbidly obese children, compared with overweight children, indicates limited early identification of child weight issues or the possibility that child weight management programmes such as $\mathrm{PEACH}$ may have been considered a last resort, rather than first step, for families who have attempted to manage their child's weight. A previous qualitative study, which explored reasons why a sample of twenty-one parents enrolled their children in PEACH QLD, reported that parents were aware of their child's weight issue 
for a long time and their enrolment came after previous unsuccessful attempts at weight management and was triggered by their child's own concern with their weight ${ }^{(40)}$.

The aim of this study was to evaluate the effectiveness in the 'real-world' practice setting of an evidence-based service targeting families with obese children. The study purposefully did not intend to repeat the evaluation of the efficacy of the PEACH intervention. Inclusion of a control group was not required to achieve this aim, and furthermore, in the practice context, would not have been acceptable to programme recipients, the service providers who delivered the PEACH Program nor the funder as core business for both the funder and service providers is service delivery for the community rather than scholarly research. This means that we cannot extrapolate our results to those families who were not ready or otherwise unable to engage in the programme. Hence, it is difficult to say whether those families who did not provide data did/did not experience any success in the programme. At the first session, parents received a 105-colour-page PEACH workbook, which outlined the programme principles, content for each session and reputable sources for further information and support. Thus, parents had access to programme content, which they may have engaged with on an ad hoc, self-paced basis. Although the programme is designed to be facilitated by a trained professional and be group-based to enable peer support and shared learning, this may not have been enjoyable or acceptable for all parents and may have impacted engagement. Ultimately, we have no way of ascertaining the applicability or effect of PEACH on the group that did not report paired data. However, these are the very families who are unlikely to seek support from and engage with the kind of service that this study evaluates. It highly likely that a range of evidence-based practice/service responses will be required to meet the diverse needs of families with overweight and obese children. This study evaluates one such response and demonstrates that it was acceptable and effective in a select group of families. Clearly, studies are required that are specifically designed to identify other engagement strategies and features of intervention design and delivery to extend reach and range of effective service/practice level responses to childhood obesity. Ultimately, it is clear that for the sample who did engage in sessions and provide pre-post-programme data, PEACH was effective in achieving child and family lifestyle behaviour change and consequently a relative reduction in children's level of overweight.

\section{Strengths and limitations}

A strength of the current study is the collection of comprehensive measures for lifestyle behaviours up to 4 months after completion of the intervention, rather than immediately after the end of group sessions. As such, these evaluation data provide for a follow-up period. When delivered at a larger scale, programmes may have limited evaluation depending on needs, budget and health service. When a programme is fully translated, it becomes embedded in existing practice and health services where evaluation is likely to be curtailed and restricted to limited service monitoring data such as attendance and/or rudimentary assessment of participant satisfaction.
With the exclusion of child anthropometric measurements, which were largely collected at sessions by trained facilitators, all other evaluation data (parent self-efficacy, diet, activity) were parent-reported and may be subject to acquiescence bias. Although it was not feasible for the primary school children enrolled to provide information on their dietary intake and activity behaviours, we acknowledge that there are objective measures that could have been used in place of, or to validate, parentreported data (e.g. doubly labelled water, accelerometry). Our selection of data collection tools to assess outcomes was determined by the large sample size, evaluation budget and the needs of the funder to ascertain public health benefit - that is, data were collected with an emphasis on service provision rather than research. Therefore, our methods reflected the need to reduce participant burden and select economical methods that are feasible for use in evaluation of programmes or health services delivered at scale within the community setting.

\section{Conclusion}

When delivered on a large scale, PEACH is an effective familybased, multi-component, lifestyle weight management programme for overweight and obese children. Investment in the delivery of childhood obesity management services at scale should be informed by evidence, and the results of this study make a substantive contribution to the limited body of existing evidence. PEACH has demonstrated its effectiveness across the translation continuum. This research contributes to the limited data available on the magnitude of dietary, activity and anthropometric change, which can be expected from a programme when delivered at scale. Further research is needed to determine whether these lifestyle changes are sustainable and can be maintained in the long term following delivery at scale and the system and service factors that support such a programme being routinely available as part of universal child health as recommended by the World Health Organization ${ }^{(1)}$. Key challenges for efficient provision of childhood obesity management programmes include the identification of excess weight early in its evolution and the engagement of families in these services.

\section{Acknowledgements}

The authors acknowledge Ms D. Croyden for their leadership role across the project, and all staff from the Queensland University of Technology Implementation team and Flinders University Evaluation team who contributed to implementation and evaluation of the PEACH programme in Queensland from 2013 to 2016. The authors are grateful to all facilitators, parents and children for their participation in PEACH QLD.

PEACH Queensland was funded by the Queensland Government of Australia (2014-2016). The views expressed in this publication do not necessarily reflect those held by the Queensland Health Department or the Queensland Government of Australia. The PEACH Queensland Project was originally funded as a joint Australian, State and Territory Government initiative under the National Partnership Agreement on Preventive Health (2013-2014). 
A. M. M. and L. A. D. conceived and designed the PEACH QLD study and its evaluation framework, and selected data collection tools. L. A. D. directed the PEACH QLD Project, H. A. V. oversaw the implementation in Queensland and A. M. M. and J. M. managed the evaluation. C. J. M. managed data collection, entry and integrity, performed the statistical analysis and wrote the first draft of the manuscript. A. M. M., H. A. V., J. M. and L. A. D. assisted with data interpretation and writing the manuscript. All authors contributed to all reviews and revisions of the manuscript and have read and approved the final version.

A. M. M. and L. A. D. are inventors of the original PEACH programme and have received licence money for the use of PEACH materials in the PEACH QLD Project. All other authors declare no conflicts of interest.

\section{References}

1. Commission on Ending Childhood Obesity (2016) Report of the Commission on Ending Childhood Obesity. Geneva: World Health Organization.

2. Gicevic S, Aftosmes-Tobio A, Manganello JA, et al. (2016) Parenting and childhood obesity research: a quantitative content analysis of published research 2009-2015. Obes Rev 17, 724-734.

3. Magarey AM, Perry RA, Baur LA, et al. (2011) A parent-led family-focused treatment program for overweight children aged 5 to 9 years: the PEACH RCT. Pediatrics 127, 214-222.

4. National Health and Medical Research Council (2013) Clinical Practice Guidelines for the Management of Overweight and Obesity in Adults, Adolescents and Children in Australia. Canberra: Commonwealth of Australia.

5. Gerards SMPL, Hummel K, Dagnelie PC, et al. (2013) Parental self-efficacy in childhood overweight: validation of the Lifestyle Behavior Checklist in the Netherlands. Int J Behav Nutr Phy 10, 7.

6. Oude Luttikhuis H, Baur L, Jansen H, et al. (2009) Interventions for treating obesity in children. Cochrane Database Syst Rev, issue 1, CD001872.

7. Fagg J, Chadwick P, Cole TJ, et al. (2014) From trial to population: a study of a family-based community intervention for childhood overweight implemented at scale. Int J Obes (Lond) 38, 1343-1349.

8. Hardy LL, Mihrshahi S, Gale J, et al. (2015) Translational research: are community-based child obesity treatment programs scalable? BMC Public Health 15, 652.

9. Golley RK, Magarey AM, Baur LA, et al. (2007) Twelve-month effectiveness of a parent-led, family-focused weight-management program for prepubertal children: a randomized, controlled trial. Pediatrics 119, 517-525.

10. Golley RK, Perry RA, Magarey A, et al. (2007) Family-focused weight management program for five- to nine-year-olds incorporating parenting skills training with healthy lifestyle information to support behaviour modification. Nutr Diet 64, 144-150.

11. National Health and Medical Research Council (2013) Eat for Health: Australian Dietary Guidelines. Canberra: Department of Health and Ageing, Commonwealth of Australia.

12. Moores CJ, Miller J, Perry RA, et al. (2017) CONSORT to community: translation of an RCT to a large-scale community intervention and learnings from evaluation of the upscaled program. BMC Public Health 17, 918.

13. Croyden DL, Vidgen HA, Esdaile E, et al. (2018) A narrative account of implementation lessons learnt from the dissemination of an up-scaled state-wide child obesity management program in Australia: $\mathrm{PEACH}^{\mathrm{TM}}$ (Parenting, Eating and Activity for Child Health) Queensland. BMC Public Health 18, 347.

14. Australian Bureau of Statistics (2013) Socio-Economic Indexes for Areas (SEIFA) 2011. Canberra, ACT, Australia: Australian Bureau of Statistics.

15. University of Adelaide (2013) Accessibility/Remoteness Index of Australia (ARIA2011+), 2011+ ed. Adelaide, SA, Australia: University of Adelaide.

16. Lucas N, Nicholson JM \& Maguire B (2011) The Longitudinal Study of Australian Children Annual Statistical Report 2010. Canberra: Australian Government, Australian Institute of Family Studies.

17. Hinkin TR (1995) A review of scale development practices in the study of organizations. J Manage 21, 967-988.

18. Kuczmarski RJ, Ogden CL, Guo SS, et al. (2002) 2000 CDC Growth Charts for the United States: methods and development. Vital Health Stat 11, 1-190.

19. Freeman JV, Cole TJ, Chinn S, et al. (1995) Cross sectional stature and weight reference curves for the UK, 1990. Arch Dis Child 73, 17-24.

20. Centers for Disease Control and Prevention (2016) A SAS Program for the 2000 CDC Growth Charts (ages 0 to 20 years). https://www.cdc.gov/nccdphp/dnpao/growthcharts/resources/ sas.htm\#modalIdString_CDCTable_1 (accessed June 2017).

21. Magarey A, Golley R, Spurrier N, et al. (2009) Reliability and validity of the Children's Dietary Questionnaire; a new tool to measure children's dietary patterns. Int J Pediatr Obes 4, 257-265.

22. Golley RK, Magarey AM \& Daniels LA (2011) Children's food and activity patterns following a six-month child weight management program. Int J Pediatr Obes 6, 409-414.

23. Telford A, Salmon J, Jolley D, et al. (2004) Reliability and validity of physical activity questionnaires for children: The Children's Leisure Activities Study Survey (CLASS). Pediatr Exerc Sci 16, 64-78.

24. Department of Health (2014) Australia's Physical Activity and Sedentary Behaviour Guidelines 5-12 Years. Canberra, ACT, Australia: Commonwealth of Australia.

25. Cole TJ \& Lobstein T (2012) Extended international (IOTF) body mass index cut-offs for thinness, overweight and obesity. Pediatr Obes 7, 284-294.

26. McCarthy HD \& Ashwell M (2006) A study of central fatness using waist-to-height ratios in UK children and adolescents over two decades supports the simple message - 'keep your waist circumference to less than half your height'. Int J Obesity (Lond) 30, 988-992.

27. Berge JM \& Everts JC (2011) Family-based interventions targeting childhood obesity: a meta-analysis. Child Obes 7, 110-121.

28. Loveman E, Al-Khudairy L, Johnson RE, et al. (2015) Parentonly interventions for childhood overweight or obesity in children aged 5 to 11 years. Cochrane Database Syst Rev, issue 12, CD012008.

29. Sacher PM, Kolotourou M, Chadwick PM, et al. (2010) Randomized controlled trial of the MEND program: a familybased community intervention for childhood obesity. Obesity (Silver Spring) 18, Suppl. 1, S62-S68.

30. Okely AD (2007) Evidence-guided approaches to addressing child obesity: What approaches can dietitians use in their everyday practice? Nutr Diet 64, 140-141.

31. West F, Sanders MR, Cleghorn GJ, et al. (2010) Randomised clinical trial of a family-based lifestyle intervention for childhood obesity involving parents as the exclusive agents of change. Behav Res Ther 48, 1170-1179.

32. Moens E \& Braet C (2012) Training parents of overweight children in parenting skills: a 12-month evaluation. Behav Cogn Psychother 40, 1-18. 
33. Costanzo PR \& Woody EZ (1985) Domain-specific parenting styles and their impact on the child's development of particular deviance: the example of obesity proneness. J Social and Clin Psychol 3, 425-445.

34. Williams SL, Van Lippevelde W, Magarey A, et al. (2017) Parent engagement and attendance in PEACH QLD - an up-scaled parent-led childhood obesity program. BMC Public Health 17, 559.

35. Marmot M, \& Commission on Social Determinants of Health (2007) Achieving health equity: from root causes to fair outcomes. Lancet 370, 1153-1163.

36. Dhaliwal J, Nosworthy NM, Holt NL, et al. (2014) Attrition and the management of pediatric obesity: an integrative review. Child Obes 10, 461-473.
37. Skelton JA \& Beech BM (2011) Attrition in paediatric weight management: a review of the literature and new directions. Obes Rev 12, e273-e281.

38. Fisher L, Fraser J \& Alexander C (2006) Caregivers' inability to identify childhood adiposity: a cross-sectional survey of rural children and their caregivers' attitudes. Aust J Rural Health 14, 56-61.

39. Maynard LM, Galuska DA, Blanck HM, et al. (2003) Maternal perceptions of weight status of children. Pediatrics $\mathbf{1 1 1}$, 1226-1231

40. Davidson K \& Vidgen H (2017) Why do parents enrol in a childhood obesity management program?: a qualitative study with parents of overweight and obese children. BMC Public Health 17, 159. 